\title{
Management and outcome of recurrent adult craniopharyngiomas: an analysis of 42 cases with long-term follow-up
}

\author{
Mazda K. Turel, MCh, Georgios Tsermoulas, MD, Lior Gonen, MD, George Klironomos, MD, \\ Joao Paulo Almeida, MD, Gelareh Zadeh, MD, PhD, FRCSC, and Fred Gentili, MD, FRCSC
}

Department of Neurosurgery, Toronto Western Hospital, Toronto, Ontario, Canada

OBJECTIVE The treatment of recurrent and residual craniopharyngiomas is challenging. In this study the authors describe their experience with these tumors and make recommendations on their management.

METHODS The authors performed an observational study of adult patients ( $\geq 18$ years) with recurrent or residual craniopharyngiomas that were managed at their tertiary center. Retrospective data were collected on demographics and clinical, imaging, and treatment characteristics from patients who had a minimum 2-year follow-up. Descriptive statistics were used and the data were analyzed.

RESULTS There were 42 patients ( 27 male, 15 female) with a mean age of $46.3 \pm 14.3$ years. The average tumor size was $3.1 \pm 1.1 \mathrm{~cm}$. The average time to first recurrence was $3.6 \pm 5.5$ years (range $0.2-27$ years). One in 5 patients (8/42) with residual/recurrent tumors did not require any active treatment. Of the 34 patients who underwent repeat treatment, $12(35.3 \%)$ had surgery only (transcranial, endoscopic, or both), 9 (26.5\%) underwent surgery followed by adjuvant radiation therapy (RT), and $13(38.2 \%)$ received RT alone. Eighty-six percent $(18 / 21)$ had a gross-total $(n=4)$ or near-total (n $=14$ ) resection of the recurrent/residual tumors and had good local control at last follow-up. One of 5 patients $(7 / 34)$ who underwent repeat treatment had further treatment for a second recurrence. The total duration of follow-up was $8.6 \pm 7.1$ years. The average Karnofsky Performance Scale score at last follow-up was 80 (range 40-90). There was 1 death.

CONCLUSIONS Based on this experience and in the absence of guidelines, the authors recommend an individualized approach for the treatment of symptomatic or growing tumors. This study has shown that 1 in 5 patients does not require repeat treatment of their recurrent/residual disease and can be managed with a "scan and watch" approach. On the other hand, 1 in 5 patients who had repeat treatment for their recurrence in the form of surgery and/or radiation will require further additional treatment. More studies are needed to best characterize these patients and predict the natural history of this disease and response to treatment.

https://thejns.org/doi/abs/10.3171/2016.9.FOCUS16315

KEY WORDS adult; craniopharyngioma; endoscopy; radiation; recurrent; residual; surgery

$\mathrm{C}$ RANIOPHARYNGIOMA is a dysembryogenetic benign tumor in a "malignant" location with an unpredictable growth pattern and a tendency to invade critical neurovascular structures. ${ }^{31}$ These tumors account for less than $5 \%$ of all intracranial tumors. ${ }^{3}$ While the treatment of primary tumors is challenging, the management of recurrent tumors is even more difficult and complex. Repeat surgery is often associated with an increased risk for complications, including a higher rate of permanent morbidity as well as death., ${ }^{4,11,20,22,23}$ There are currently no established guidelines for the optimal timing of repeat surgery versus radiation therapy (RT). Likewise, there is no clear consensus on the optimal management of any residual tumor. In addition, because this tumor is more common in the pediatric population, there is limited adult literature on the management of recurrent tumors. While several factors such as incomplete resection, adherence to critical neurovascular structures, and lack of adjuvant RT have been purported to be risk factors for recurrence, there is a paucity of data in the literature on the optimal manage-

ABBREVIATIONS EEA = endoscopic endonasal approach; GTR = gross-total resection; KPS = Karnofsky Performance Scale; NTR = near-total resection; RT = radiation therapy; STR = subtotal resection. 
ment of recurrent craniopharyngiomas in adults using a multidisciplinary approach. ${ }^{26}$

The endoscopic endonasal approach (EEA) has expanded the treatment options for this condition with favorable results..$^{5-7}$ Unfortunately, the biological aggressiveness of this tumor-with its tendency to recur and invade vital adjacent structures such as the pituitary stalk, hypothalamus, and third ventricle and perforating blood vessels-continues to add to the dilemmas in management. The choice of retreatment can have a significant impact on the visual, nutritional, hormonal, and neuropsychological outcome of the patient with a significant alteration of their quality of life.

In this study we review the outcomes following management of recurrent and residual craniopharyngiomas in adults, outline the trials and tribulations of treating this condition at our center, and evaluate whether a uniform treatment algorithm could be proposed for the management of these challenging tumors. We also review the pertinent literature on the current principles regarding the management of recurrent craniopharyngiomas.

\section{Methods \\ Patient Population}

Between January 2002 and December 2013, all adult patients ( $\geq 18$ years of age) who were treated for a recurrent or a residual craniopharyngioma at our institute were included. The study was approved by the Institutional Review Board of Toronto Western Hospital. The term "recurrent tumor" refers to a tumor with documented growth on serial imaging after surgery and/or radiation therapy. Residual tumor refers to the presence of obvious tumor after surgery but no demonstration of significant growth on serial imaging.

All patients underwent preoperative MRI at the time of presentation to us with recurrent/residual tumor. Tumor size was calculated in centimeters using the single largest diameter. Preoperative clinical and radiological data were entered retrospectively into a Microsoft Excel database from the electronic patient records of the hospital. All patients underwent preoperative and postoperative visual field and endocrinological evaluations. Impaired visual acuity was defined as acuity of less than 20/20 in either eye. The final outcome was assessed using the Karnofsky Performance Scale (KPS) score.

\section{Treatment Policy}

At our center, patients are evaluated by a multidisciplinary team that consists of a skull base neurosurgeon, endocrinologist, neuroradiologist, radiation oncologist, and sometimes a medical oncologist, and decisions regarding the management of the recurrent craniopharyngioma are made in a collaborative manner, which includes input from the patient. Updated radiological findings, endocrine status, visual performance, and overall neurological status are all considered crucial factors in these discussions. However, a strict protocol for management of recurrent craniopharyngiomas was not established prior to the study.

The common policy was to treat patients with symptomatic disease, or those with tumors with a growth rate expected to cause symptoms during the patient's expected lifetime. Both transcranial and endoscopic approaches were used based on tumor location and extension. The pterional or the interhemispheric transbasal or transcallosal routes were the most preferred open approaches. However, over the last several years, there has been an increased use of expanded EEA techniques in the management of recurrent tumors. The EEA for these tumors has been described in several previous publications. ${ }^{5,7-9,12,17}$

Image guidance (Medtronic/Stryker) was used in all surgeries. This technology is particularly useful in patients who have previously undergone operations using the transsphenoidal approach in whom the surgical corridor has already been violated, and therefore the classic landmarks (sellar floor, clival indentation, carotid and optic nerve protuberances, and the opticocarotid recess) are not easy to identify and can help the surgeon to maintain orientation even in the presence of distorted anatomy. ${ }^{6,7}$ The micro-Doppler probe is also routinely used for carotid localization. RT was usually reserved for tumors that had demonstrated growth on serial imaging after 1 or more surgical procedures for the recurrence, and when it was believed that further intervention would carry significant risk or not be beneficial. In some patients, if it was believed that repeat surgery would not be feasible, upfront RT was considered. All patients who underwent RT had fractionated stereotactic radiosurgery.

\section{Follow-Up}

Patients were routinely followed up with a CT scan on the day after surgery and then at 6 weeks with MRI unless further imaging was warranted between these time periods. MRI was then performed at 3, 6, and 12 months in the first year and then biannually thereafter. This schedule was also modified based on individual patient and tumor characteristics. If the tumor appeared stable over a few years, the duration of follow-up was lengthened to once a year.

\section{Results}

\section{Demographics, Radiology, and Pathology}

The cohort comprised 42 patients ( 27 male, 15 female) with a mean age of $46.3 \pm 14.3$ years (range 18-70 years; Table 1). The average tumor size at treatment of first recurrence was $3.1 \pm 1.1 \mathrm{~cm}$ (range $1-7 \mathrm{~cm}$ ). Thirty-seven patients $(88.1 \%)$ had heterogeneous lesions with a solid and cystic component within their tumor. Two patients (4.8\%) had entirely cystic recurrences and 3 patients (7.1\%) had entirely solid recurrent tumors. While in all patients the residue was within the suprasellar cistern, in an additional 11 patients $(26.2 \%)$ it was within the third ventricle and 2 patients $(4.8 \%)$ had an extension into the middle/posterior fossa. The pathology at primary surgery was adamantinomatous in 31 patients (73.8\%) and papillary in 11 (26.2\%).

\section{Clinical Findings}

Visual dysfunction was present in $64.3 \%$ (27 patients), headache in $21.4 \%$ (9 patients), weight gain in $7.1 \%$ (3 patients), and new endocrine deficit was present in $33.3 \%$ (14 patients; Table 1). 
TABLE 1. Clinical characteristics of adult patients with recurrent and residual craniopharyngiomas

\begin{tabular}{|c|c|}
\hline Variable & Value (\%) \\
\hline No. of patients & 42 \\
\hline Males/females & $27(64.3) / 15(35.7)$ \\
\hline \multicolumn{2}{|l|}{ Age (yrs) } \\
\hline Mean \pm SD & $46.3 \pm 14.3$ \\
\hline Range & $18-70$ \\
\hline \multicolumn{2}{|l|}{ Tumor size at 1st recurrence $(\mathrm{cm})$} \\
\hline Mean \pm SD & $3.1 \pm 1.1$ \\
\hline Range & $1-7$ \\
\hline \multicolumn{2}{|l|}{ Time to 1st recurrence (yrs) } \\
\hline Mean \pm SD & $3.6 \pm 5.5$ \\
\hline Range & $0.2-27$ \\
\hline \multicolumn{2}{|l|}{ Duration of follow-up (yrs) } \\
\hline Mean \pm SD & $8.6 \pm 7.1$ \\
\hline Range & $2-40$ \\
\hline \multicolumn{2}{|l|}{ Tumor consistency } \\
\hline Solid \& cystic & $37(88.1)$ \\
\hline Purely solid & $3(7.1)$ \\
\hline Purely cystic & $2(4.8)$ \\
\hline \multicolumn{2}{|l|}{ Tumor location } \\
\hline Suprasellar & $42(100)$ \\
\hline Suprasellar \& 3rd ventricle & $11(26.2)$ \\
\hline Suprasellar \& middle/posterior fossa & $2(4.8)$ \\
\hline \multicolumn{2}{|l|}{ Clinical features } \\
\hline Visual dysfunction & $27(64.3)$ \\
\hline Headache & $9(21.4)$ \\
\hline Weight gain & $3(7.1)$ \\
\hline New endocrine deficit & $14(33.3)$ \\
\hline
\end{tabular}

\section{Visual Outcomes}

Of the 27 patients with visual decline, 23 had visual acuity deficits and 16 had visual field defects. Of these 16 patients, 7 had a bitemporal hemianopia, 4 had a homonymous hemianopia, and 5 had a unilateral temporal field defect. Four patients had a visual field deficit with normal visual acuity. Of the 23 patients with visual acuity involvement, 5 patients had vision limited to perception of hand movements close to their face or perception of light alone.

After treatment at final follow-up, vision had improved in more than half the cohort (22 patients, $52.4 \%$ ) and remained the same in 19 patients $(45.2 \%)$, with only 1 patient demonstrating worsening of vision in a delayed fashion, probably related to radiation-induced vasculopathy (Table 2). Of the 22 patients who improved, 8 had complete normalization of their visual field defects. The remaining patients showed varying degrees of improvement on visual field charting. Six patients demonstrated a complete resolution of visual acuity deficit from previously impaired to $20 / 20$ vision. Of the 5 patients who had vision limited to perception of hand movements close to their face or perception of light alone, 3 of them improved to a functional
TABLE 2. Treatment paradigms of 42 adult patients with recurrent craniopharyngiomas

\begin{tabular}{|c|c|}
\hline Treatment & Value (\%) \\
\hline Surgery/radiation & $34(81)$ \\
\hline \multicolumn{2}{|l|}{ No. of recurrences* } \\
\hline 1 & 19 \\
\hline 2 & 9 \\
\hline 3 & 4 \\
\hline 4 & 1 \\
\hline 6 & 1 \\
\hline Mean no. of recurrences & 1.7 \\
\hline Surgery alone & $12 / 34(35.3)$ \\
\hline Surgery + upfront RT & $22 / 34(64.7)$ \\
\hline RT at delayed recurrence & $13 / 34(38.2)$ \\
\hline CSF diversion & $5 / 34(14.7)$ \\
\hline Retreatment after surgery \& RT & $7 / 34(20.6)$ \\
\hline Watchful observation & $8(19)$ \\
\hline \multicolumn{2}{|l|}{ Complications of treatment } \\
\hline Postop intracranial hemorrhage & 3 \\
\hline CSF leak & 4 \\
\hline Acute hydrocephalus on radiation & 1 \\
\hline Delayed epistaxis & 1 \\
\hline Mortality & 1 \\
\hline \multicolumn{2}{|l|}{ Visual outcomes at last follow-up } \\
\hline Same & $19(45.2)$ \\
\hline Improved & $22(52.4)$ \\
\hline Worse & $1(2.4)$ \\
\hline New endocrine deficit at last follow-up & 15/34 (44.1) \\
\hline Mean KPS score at last follow-up (range) & $80(40-90)$ \\
\hline
\end{tabular}

visual acuity varying from 20/30 to 20/70. The 2 patients with light perception alone remained unchanged.

\section{Endocrine Outcomes}

Fourteen patients were receiving 1 or more hormonal replacements prior to treatment. Eight patients were receiving cortisol replacement, 5 patients were prescribed thyroxine, 2 were taking oral desmopressin, and 1 patient was taking testosterone. Four patients were receiving more than 1 hormonal substitute. In addition, 15 (44.1\%) of the 34 patients who were treated had developed new endocrine deficits after surgery/RT at last follow-up. All 15 patients were taking cortisol and thyroid replacement, 12 patients were receiving gonadotropic replacements, and 11 patients were taking desmopressin. Thus, 11 (73.3\%) of the 15 patients had new panhypopituitarism at last follow-up. None of the patients with a preoperative endocrine deficit could discontinue the hormonal substitute at follow-up.

\section{Treatment Paradigms}

Of the 42 patients with recurrent/residual tumors, 34 $(81 \%)$ were treated and 8 did not have any treatment at a mean follow-up of $8.3 \pm 3.9$ years (range 5-18 years). Of 
these 34 patients treated, the recurrence was asymptomatic in 5 and symptomatic in 29 patients. All of the untreated patients experienced asymptomatic recurrences.

Of the 34 patients, 19 patients had 1 recurrence, 9 patients had 2 recurrences, 4 patients had 3 recurrences, 1 patient had 4 recurrences, and 1 patient had 6 recurrences (Table 2). The average number of recurrences per patient was 1.7.

The treatment of the recurrent tumor was surgery alone ( 1 or more surgeries) in $12(35.2 \%)$ of 34 patients (Table 2$)$ : open surgery in 1 patient, EEA in 8 patients, and open and EEA in 3 patients. Twenty-two patients $(64.7 \%)$ received RT for their recurrent tumor. Nine patients had planned surgery (4 open, 5 EEA) followed by immediate RT for their recurrence. Five of these patients had undergone a previous operation for their recurrent tumor. Thirteen patients underwent RT when their tumors recurred, either when they presented to us or when tumor grew despite surgery and/or $\mathrm{RT}$ for recurrence. Five patients required 1 or multiple CSF diversion procedures at the time of recurrence in the form of endoscopic third ventriculostomy or shunt placement.

\section{Extent of Resection and Retreatment}

Eighty-six percent $(18 / 21)$ of patients underwent a gross-total (GTR; $n=4)$ or near-total (NTR; $n=14)$ resection of the recurrent/residual tumors and had good local control at last follow-up. Seven (20.6\%) of 34 patients required additional treatment after their surgery for recurrence and RT. Of these 7 patients, 5 underwent repeat surgery (EEA alone), 1 had repeat RT alone, and 1 required repeat surgery (EEA and open) as well as RT.

\section{Complications}

Three patients experienced postoperative hemorrhage after repeat transcranial surgery ( 2 extradural hematomas, 1 subdural hematoma). All required surgical evacuation of the hematoma. Three patients suffered postoperative CSF leaks after EEA, 1 of whom required 2 repair procedures. The overall CSF leak rate was $11.8 \%$ (4/34; Table 2). One patient developed acute hydrocephalus while undergoing RT and 1 patient suffered a postoperative pulmonary embolism with an uneventful recovery. One patient experienced delayed postoperative epistaxis that was managed nonoperatively with nasal packing. There was no vascular injury to the internal carotid artery or its branches or perforators. There was 1 death in a 51-year-old man who had a transcranial operation for removal of the craniopharyngioma followed by multiple shunt revisions for recurrent hydrocephalus. He then developed a brain abscess, which was treated, and suffered a cardiac arrest a few days after an endoscopic excision of his next recurrence.

\section{Follow-Up}

The average time to first recurrence was $3.6 \pm 5.5$ years (range $0.2-27$ years). The total duration of follow-up was $8.6 \pm 7.1$ years (range $2-40$ years). The average KPS score at last follow-up was 80 (range 40-90; Table 2).

\section{Discussion}

The recurrence rate of craniopharyngiomas is reported as $0 \%-62 \%$ at 10 years, even after apparent GTR. ${ }^{14,16,23}$ The challenges encountered in the management of recurrent and residual craniopharyngiomas have resulted in various treatment options being used for these tumors. Several therapeutic modalities such as RT, stereotactic catheter placement, intratumoral bleomycin, intracystic radiotherapy, and more recently, targeted chemotherapy in patients with $B R A F$ mutations have been employed to obtain tumor control. ${ }^{1,2,13,24,27,30}$ Despite these options, there is currently no consensus concerning the optimal treatment for tumor recurrence.

\section{Craniotomy and Cranial Endoscopy in Recurrent Tumors}

Reported rates of GTR following a craniotomy for recurrent craniopharyngiomas range from $0 \%$ to $56 \%$ and are often less than $25 \%$, with increased perioperative mortality ranging from $11 \%$ to $24 \% .^{11,14,15,32}$ Microsurgery for recurrent lesions is generally acknowledged by most surgeons as more difficult than primary surgery. This is often attributed to the loss of arachnoidal planes with resultant scarring and adhesions to critical neurovascular structures from the previous surgical manipulation. The lack of arachnoidal planes, or gliotic reaction between the tumor and the normal tissue that forms a dissection cleavage plane with the hypothalamus, makes dissection more difficult with increased risk for injury to these vital anatomical structures that can result in severe postoperative morbidity. Likewise, the dense adherence of tumor to major vessels or smaller penetrating arteries much more common in recurrent lesions makes repeat surgery more difficult, reducing the rate of resection and increasing the risk for postoperative morbidity.

Thus, we believe that the decisions regarding the first modality of treatment or what is performed at the time of the first operation is very important. Most of the patients were referred to us from other neurosurgeons. It is, however, very important and most authors would agree that in craniopharyngiomas, like some other entities including chordomas, the first operation offers the best and in some cases the only opportunity to obtain a total removal of the lesion. Better education of all individuals coping with this disease would be helpful. We believe that the management of these uncommon challenging lesions should only be conducted at centers with experience and expertise.

While the majority of our recurrent cases were operated on using the EEA, craniotomy has a distinct role in addressing recurrences, including lesions within the middle and posterior cranial fossa and those restricted entirely within the third ventricle. Of the open approaches, we prefer the interhemispheric transbasal or transcallosal approach for those lesions restricted entirely within the third ventricle. Likewise, transcranial endoscopy is an important adjunct used to perform third ventriculostomies, insert Ommaya reservoirs, and fenestrate supratentorial cysts, and should be an important tool in the hands of surgeons treating this pathology.

\section{Expanded EEA in Recurrent Tumors}

The endonasal route is an important novel therapeutic option for recurrent tumors, especially when the previous 
surgery was transcranial. It serves as an untouched route and direct inferior access to the chiasm, pituitary stalk, and third ventricle, including tumors with retrosellar interpeduncular extensions that can be difficult to reach using standard open approaches. ${ }^{6,10,18,31}$ It offers the advantage of facing the tumor immediately after the dural opening without brain retraction, and optimizes visualization of the relevant anatomy through a straight surgical trajectory. ${ }^{17}$ The EEA is also particularly useful in the surgical management of tumors with residual lesions and those that have regrown from these areas that were presumably not reached or manipulated during the first transcranial surgery. ${ }^{6}$

In our opinion, lesions with extensions lateral to the internal carotid artery into the middle cranial fossa, and those with vascular encasement, are contraindications to using the EEA unless the goal of surgery is only decompression of the optic apparatus with planned adjuvant therapy for the residual tumor. Even in tumors that have been operated on previously through the EEA, the nasoseptal flap can be taken down with ease for reuse after repeat surgery. If, for any reason, this does not work, there are several other options through the nasal cavity for skull base reconstruction. . $^{25,33,34}$

In approximately one-third of our patients, surgery alone was sufficient to manage recurrent tumors that were stable during long-term follow-up after either a GTR or NTR. These patients did not receive RT or any other alternative therapy. We agree with the philosophy of Dhandapani et al., ${ }^{10}$ who stated when the goal of surgery is GTR but GTR is not achieved during the first operation, the patient should be offered another reoperation via the EEA by an experienced surgeon if feasible. RT should be used only if this second attempt at GTR is unsuccessful. Their GTR and NTR rates were $80 \%$ and $100 \%$, respectively, much higher than those described in the current literature. They also reported that reoperation via the EEA results in a similar extent of resection, visual outcome, and improvement in quality of life as that of first-time operations. Prior RT, however, did appear to decrease the extent of resection in this cohort.

\section{Management of Cystic Recurrences}

Cystic recurrences are another entity that is more favorably addressed using an endoscopic approach. Injecting bleomycin into the cyst cavity has fallen out of favor due to the side effects associated with this drug. The technique of endoscopic fenestration and drainage of cysts with removal of most of the capsule often results in prevention of cyst re-accumulation. However, in refractory cases, Solanki et al. ${ }^{29}$ have described a technique for the persistent external drainage of intractable cystic recurrences with the creation of a fistula between the cyst wall epithelium and epithelium on the nasal cavity, using a pedicled nasoseptal flap as a conduit. This is possible, however, only in subdiaphragmatic tumors with no CSF leak. Two of our patients required an Ommaya reservoir and 1 patient needed a cystoperitoneal shunt (Fig. 1).

We also managed 2 patients who had similar cystic recurrences but whose cases contrasted in other details. In one of these patients, the cyst was observed over several years and eventually showed almost complete resolution (Fig. 2); in the other patient, there was massive re-accumu-
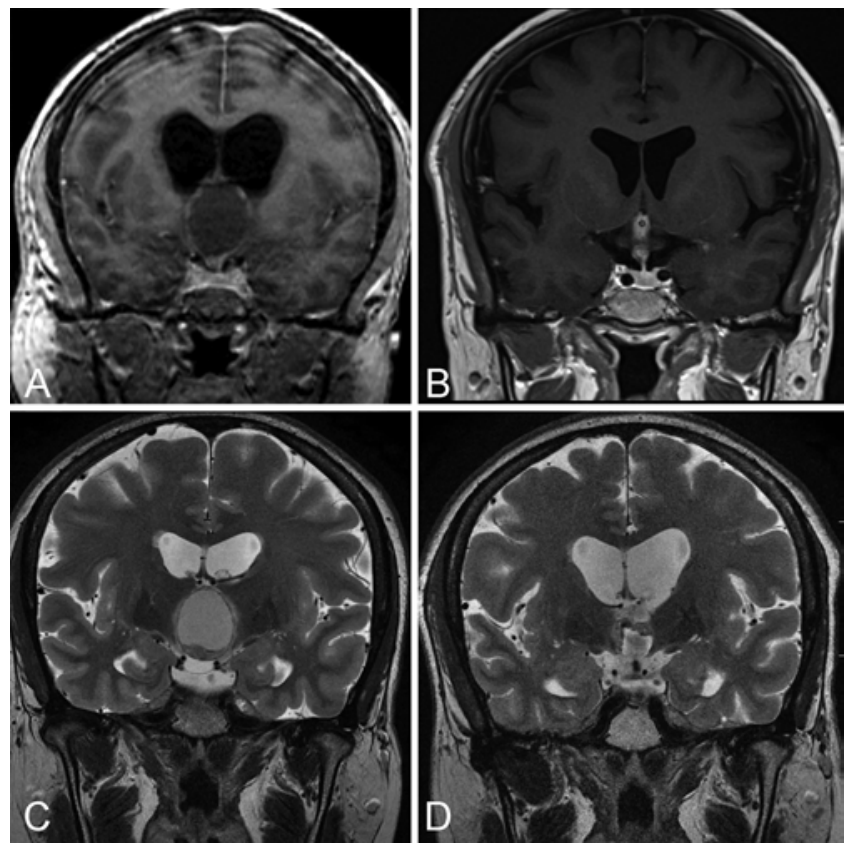

FIG. 1. Coronal MR images of a 45-year-old man with a suprasellar and third-ventricle craniopharyngioma who underwent an interhemispheric transcallosal approach with NTR. A: Preoperative T1-weighted Gd-enhanced image. B: Postoperative T1-weighted MR image at 1 year showing a well-decompressed cyst with the Ommaya reservoir in situ. C: Two-year postoperative T2-weighted image showing reaccumulation of the cyst, for which he underwent a repeat transcranial operation with reinsertion of the Ommaya reservoir. D: One-year postoperative T2-weighted image after the second surgery showing a stable lesion, thus eliminating the need for radiation thus far.

lation of the residual cyst within 6 months of follow-up that required transcranial surgery followed by radiation (Fig. 3).

\section{Radiation in the Management of Recurrent Tumors}

Many authors have reported on the favorable outcome of radiation following maximal safe resection of craniopharyngiomas. Some authors have suggested that subtotal resection (STR) and RT may provide superior clinical outcome, achieving better disease control than GTR or STR, and can limit the adverse effects associated with aggressive resection. ${ }^{28}$ However, RT may lead to long-term problems such as vasculopathies, intellectual deficits, and secondary tumors, a problem more concerning in the pediatric population than in adults. In addition, patients who underwent GTR had an increased rate of long-term endocrine deficits compared with those undergoing STR, and those undergoing STR as well as RT. Other studies report conflicting results, with lower control rates following RT for recurrent tumors, requiring multiple treatments. ${ }^{14,19}$ These data are mostly limited to the pediatric population. In adults with a mean age of 30 years, the recurrence rate after radiosurgery was as high as $45 \%$ at 4 years in 1 study. ${ }^{21}$

\section{Retreatment After Surgery and RT for Multiple Recurrences}

The EEA served as a workhorse approach in patients 


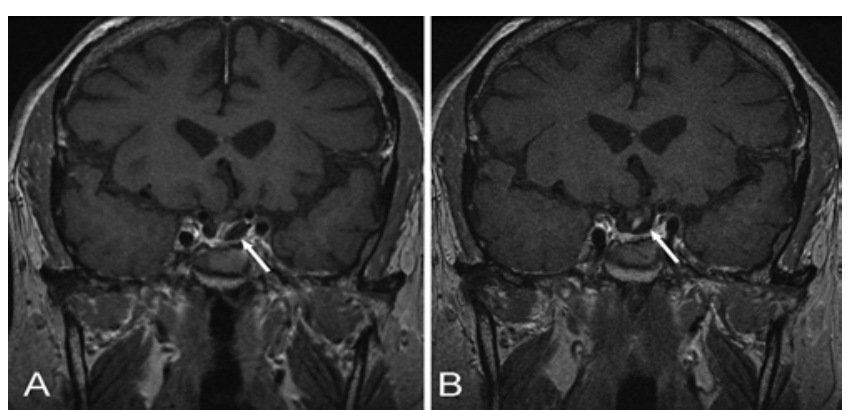

FIG. 2. Coronal T1-weighted, Gd-enhanced MR images of a 60 -year-old woman obtained 4 years after an endoscopic endonasal excision of a craniopharyngioma, showing a stable cystic enhancing lesion (arrow) in the left sellar region $(\mathbf{A})$ that demonstrated almost complete resolution (arrow) with watchful waiting 2 years later (B).

who experienced tumor recurrence after undergoing surgery (both transcranial and endoscopic) and radiation for their recurrent tumors. This approach was used in $85 \%$ of the cases, either as a standalone (5/7) or in combination with a transcranial approach (6/7) for effective long-term control (Figs. 4 and 5). In one other patient long-term control was achieved with repeat radiation alone. The senior author (F.G.) believes that tumors do recur despite GTR if the stalk is preserved, and one strategy to eliminate tumor recurrence is complete removal of the stalk with the tumor. Whether this strategy should be attempted only in patients who have preoperative endocrine dysfunction, or should be considered a valid option, even in patients with normal hormonal status, is controversial. We offer this option to our patients with recurrent tumors, enabling them to weigh the risks and benefits of lifelong hormonal replacement versus repeated tumor recurrence. The fact that a small percentage of patients develop panhypopituitarism even with preservation of the stalk complicates the issue even further. A very detailed discussion occurs with patients who are considering repeat surgery for these tumors, to provide these patients with the best outcome while taking into consideration their expectations from surgery and quality of life issues.

\section{Residual Tumors With No Growth}

The recurrence rate after STR varies from $30 \%$ to $100 \%$ with an average of $65 \%$. With the addition of RT, this rate drops to $0 \%-30 \%$, with an average of $17 \%$. Considering these data, one can confirm that tumor recurrence is common and that tumor growth from residual disease can be expected. However, in 8 (19\%) of 42 patients the residual tumor was followed up without any intervention for a mean duration of $8.3 \pm 3.9$ years (range 5-18 years). Hence, it is imperative to understand that further treatment in residual tumors should only be reserved for tumors with documented growth, as there are no conclusive factors that help predict recurrence. The presence of large chunky calcification may indicate inert tissue and may not warrant any further treatment, despite its sometimes worrisome appearance. The biological behavior of craniopharyngiomas varies from patient to patient; while some remain stable, others grow during an erratic period of time. Mo-

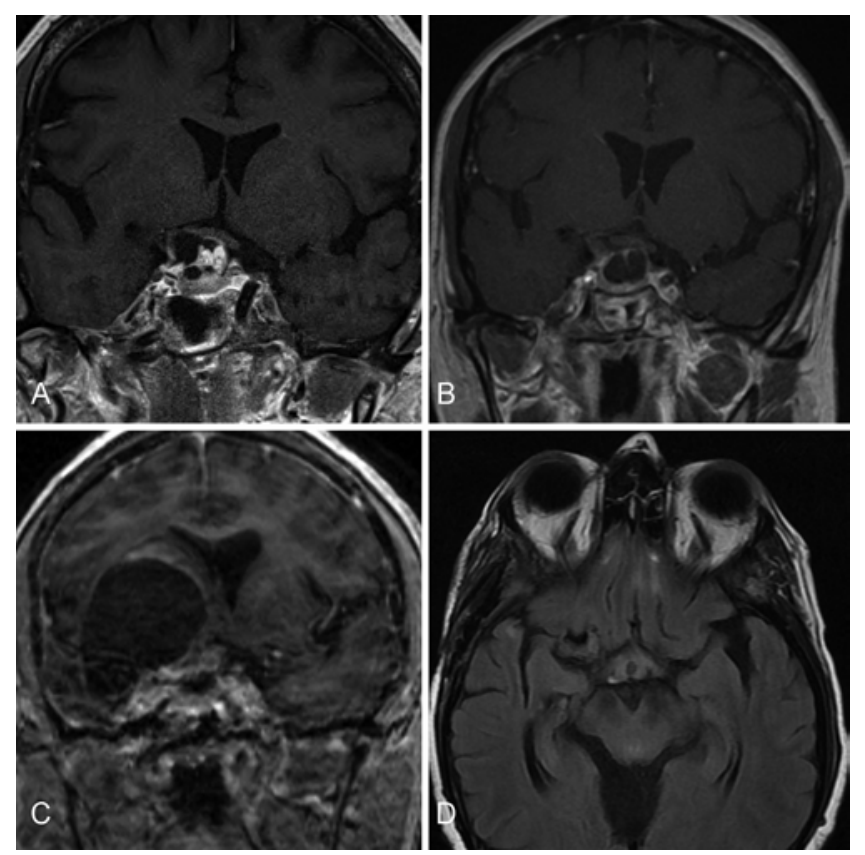

FIG. 3. A: T1-weighted Gd-enhanced coronal MR image of a 63-yearold woman showing a solid and cystic suprasellar craniopharyngioma for which she underwent an endoscopic endonasal NTR with preservation of the pituitary stalk. B: One-year postoperative coronal, T1-weighted, Gd-enhanced MR image showing an asymptomatic residual suprasellar cystic enhancing lesion. C: Coronal T1-weighted, Gd-enhanced MR image showing massive cystic accumulation of the recurrence within 6 months requiring urgent surgery via a pterional approach followed by RT. D: Axial T2-weighted FLAIR image showing a stable lesion 3 months after radiation. The clinical course in this patient is significantly different from the patient in Fig. 2 in which a similar cyst regressed with time, adding further difficulty to the management of these tumors.

lecular analysis may provide further understanding of the behavior of these tumors and help guide adoption of strategies based on the genetic profile of craniopharyngiomas. While the results are preliminary, recent reports of agents targeting the $B R A F$ mutation have shown promise in controlling tumor growth.,

\section{Limitations of the Study}

The study is limited by its retrospective nature. Because several patients underwent operations elsewhere at different institutions many years ago, their primary medical records were not available to analyze factors that could predict recurrence. Information on infiltration of the hypothalamus and adherence to the carotid artery was not available. Due to the heterogeneity of the cases and variability of the treatment options, there was no standardized protocol for the management of recurrences. In view of the limited number of patients and with almost half having more than 1 recurrence, analysis of factors determining outcomes of treatment was also not possible. Detailed molecular analysis such as investigation of $B R A F$ mutations was not performed on all patients to better understand the molecular profile, and to elucidate if this could contribute to recurrence. We also could not provide data on their hypothalamic dysfunction or detailed cognitive 

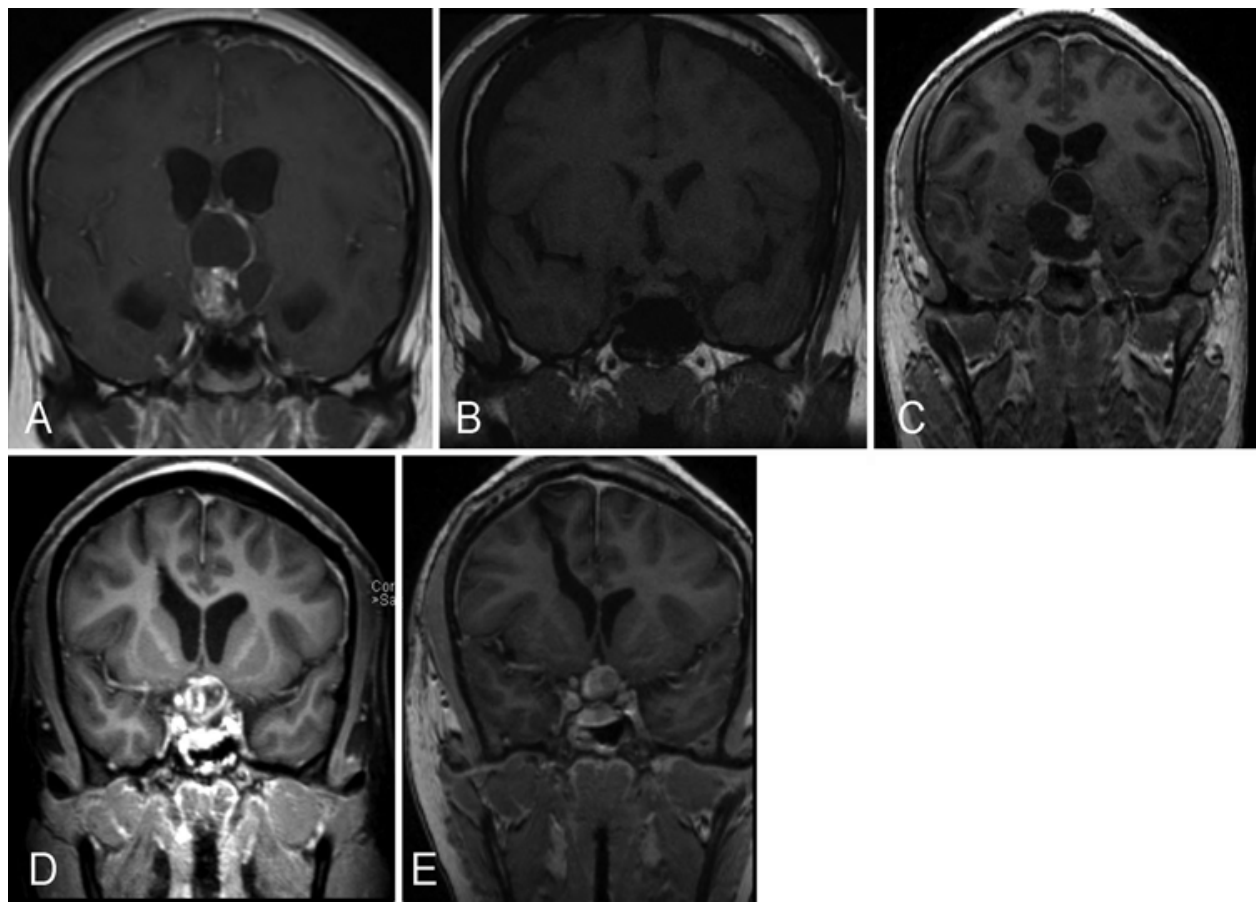

FIG. 4. Coronal T1-weighted plain (B) and Gd-enhanced (A, C-E) MR images of an 18-year-old girl who underwent transcranial NTR of a craniopharyngioma. A: Preoperative image. B: Six-month postoperative image showing a good resection. C: Image showing recurrence of the lesion 1 year after surgery. D: Image demonstrating that the patient underwent endoscopic excision for the tumor followed by RT 6 months later for further recurrence. E: Image showing that the patient experienced another recurrence despite this additional treatment and underwent repeat endoscopic surgery and a total excision with resection of the stalk. Afterwards, there was no recurrence at the 5 -year follow-up. This patient provides evidence that the pituitary stalk may have to be resected in cases of multiple recurrences.

outcomes, quality of life, or subjective or objective sinonasal outcomes in recurrent cases. Nonetheless, the KPS score (Fig. 6) does provide a relatively good measure of the patients' general well-being and activities of daily living. However, this study does shed light on the added value of endoscopy for the management of recurrences and adds credence to emerging literature that this should be attempted as a first line of treatment for the management of recurrence, instead of upfront RT, as it could prevent RT in about a quarter of the patients. The study also provides a descriptive analysis (with figures) of our attempts at management of these complex lesions, drawing some important conclusions. The long follow-up duration further adds to the understanding and natural history of these lesions after treatment.

\section{Conclusions}

Due to the variability of the nature of recurrent tumors, irrespective of the surgical approach and/or the administration of RT, treatment of adults with recurrent craniopharyngiomas should be tailored on a case-by-case basis.
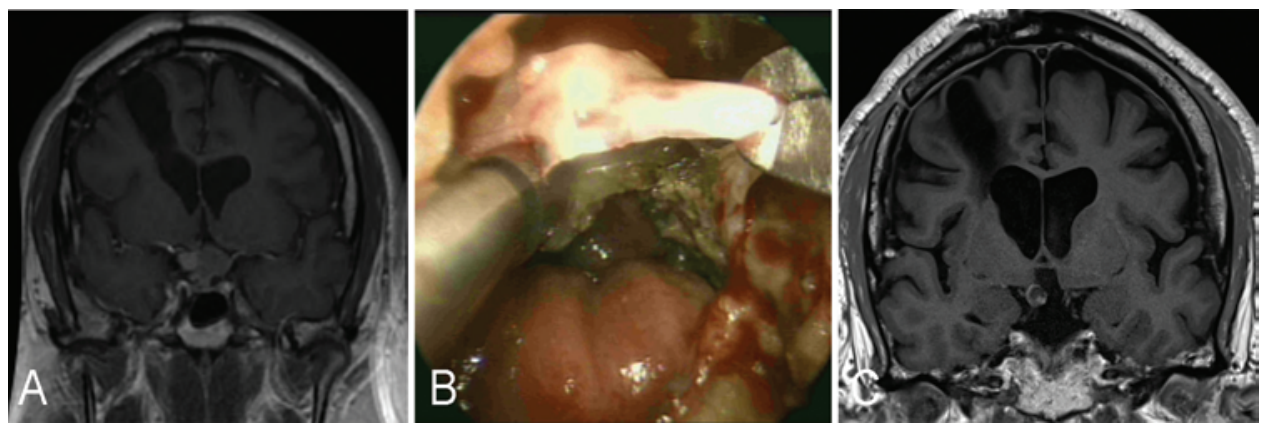

FIG. 5. This 46-year-old man underwent a transcranial surgery elsewhere followed by radiation for the craniopharyngioma and presented to our institute 3 years later with a recurrence. A: Coronal preoperative T1-weighted, Gd-enhanced MR image showing the residual craniopharyngioma. B: Intraoperative endoscopic image showing an NTR. C: Six-year postoperative coronal T1weighted plain MR image demonstrating a very small, stable, suprasellar residue that has remained unchanged in size since the time of the repeat operation, demonstrating the value of endoscopic surgery following transcranial surgery, as well as RT. 


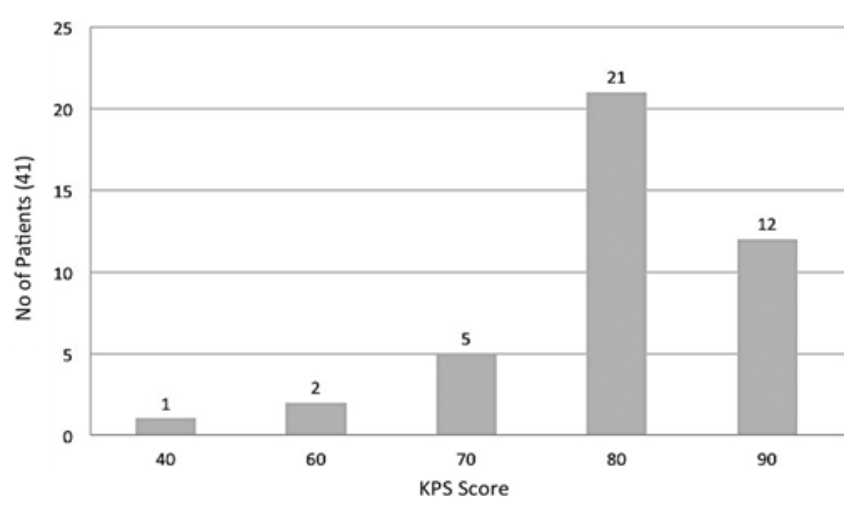

FIG. 6. Summary of KPS scores of all patients at last follow-up.

This study, however, does demonstrate the superiority of the EEA in the management of recurrent tumors and can be used as a standalone option in a third of the cases to obtain long-term control. This study also shows the benefit of the EEA in patients with recurrences after multiple surgeries and RT. Another interesting feature of this study is that a small subset of residual tumors can be managed long-term with observation alone, without the need for any adjuvant therapy.

\section{References}

1. Brastianos PK, Santagata S: Endocrine tumors: BRAF V600E mutations in papillary craniopharyngioma. Eur J Endocrinol 174:R139-R144, 2016

2. Brastianos PK, Taylor-Weiner A, Manley PE, Jones RT, DiasSantagata D, Thorner AR, et al: Exome sequencing identifies BRAF mutations in papillary craniopharyngiomas. Nat Genet 46:161-165, 2014

3. Bunin GR, Surawicz TS, Witman PA, Preston-Martin S, Davis F, Bruner JM: The descriptive epidemiology of craniopharyngioma. Neurosurg Focus 3(6):e1, 1997

4. Caldarelli M, di Rocco C, Papacci F, Colosimo C Jr: Management of recurrent craniopharyngioma. Acta Neurochir (Wien) 140:447-454, 1998

5. Cappabianca P, Cavallo LM, Esposito F, De Divitiis O, Messina A, de Divitiis E: Extended endoscopic endonasal approach to the midline skull base: the evolving role of transsphenoidal surgery. Adv Tech Stand Neurosurg 33:151-199, 2008

6. Cavallo LM, Prevedello DM, Solari D, Gardner PA, Esposito F, Snyderman CH, et al: Extended endoscopic endonasal transsphenoidal approach for residual or recurrent craniopharyngiomas. J Neurosurg 111:578-589, 2009

7. Cavallo LM, Frank G, Cappabianca P, Solari D, Mazzatenta D, Villa A, et al: The endoscopic endonasal approach for the management of craniopharyngiomas: a series of 103 patients. J Neurosurg 121:100-113, 2014

8. de Divitiis E, Cappabianca P, Cavallo LM, Esposito F, de Divitiis O, Messina A: Extended endoscopic transsphenoidal approach for extrasellar craniopharyngiomas. Neurosurgery 61 (5 Suppl 2):219-228, 2007

9. de Divitiis E, Cavallo LM, Cappabianca P, Esposito F: Extended endoscopic endonasal transsphenoidal approach for the removal of suprasellar tumors: part 2. Neurosurgery 60:46-59, 2007

10. Dhandapani S, Singh H, Negm HM, Cohen S, Souweidane MM, Greenfield JP, et al: Endonasal endoscopic reoperation for residual or recurrent craniopharyngiomas.
J Neurosurg [epub ahead of print May 6, 2016. DOI: 10.3171/2016.1.JNS152238]

11. Fahlbusch R, Honegger J, Paulus W, Huk W, Buchfelder M: Surgical treatment of craniopharyngiomas: experience with 168 patients. J Neurosurg 90:237-250, 1999

12. Gardner PA, Prevedello DM, Kassam AB, Snyderman CH, Carrau RL, Mintz AH: The evolution of the endonasal approach for craniopharyngiomas. J Neurosurg 108:10431047, 2008

13. Julow J, Lányi F, Hajda M, Szeifert G, Simkovics M, Tóth $\mathrm{S}$, et al: Further experiences in the treatment of cystic craniopharyngeomas with yttrium 90 silicate colloid. Acta Neurochir Suppl (Wien) 42:113-119, 1988

14. Karavitaki N: Management of craniopharyngiomas. J Endocrinol Invest 37:219-228, 2014

15. Karavitaki N, Brufani C, Warner JT, Adams CBT, Richards $\mathrm{P}$, Ansorge $\mathrm{O}$, et al: Craniopharyngiomas in children and adults: systematic analysis of 121 cases with long-term follow-up. Clin Endocrinol (Oxf) 62:397-409, 2005

16. Karavitaki N, Cudlip S, Adams CBT, Wass JAH: Craniopharyngiomas. Endocr Rev 27:371-397, 2006

17. Kassam AB, Gardner PA, Snyderman CH, Carrau RL, Mintz AH, Prevedello DM: Expanded endonasal approach, a fully endoscopic transnasal approach for the resection of midline suprasellar craniopharyngiomas: a new classification based on the infundibulum. J Neurosurg 108:715-728, 2008

18. Kim SK, Kim YH, Park CK, Kim DG, Jung HW: Extended endoscopic endonasal approach for recurrent or residual adult craniopharyngiomas. Acta Neurochir (Wien) 156:19171922, 2014

19. Klimo P Jr, Venable GT, Boop FA, Merchant TE: Recurrent craniopharyngioma after conformal radiation in children and the burden of treatment. J Neurosurg Pediatr 15:499-505, 2015

20. Kobayashi T, Mori Y, Tsugawa T, Hashizume C, Takahashi $\mathrm{H}$ : Prognostic factors for tumor recurrence after Gamma Knife radiosurgery of partially resected and recurrent craniopharyngiomas. Nagoya J Med Sci 74:141-147, 2012

21. Lee CC, Yang HC, Chen CJ, Hung YC, Wu HM, Shiau CY, et al: Gamma Knife surgery for craniopharyngioma: report on a 20-year experience. J Neurosurg 121 Suppl:167-178, 2014

22. Minamida Y, Mikami T, Hashi K, Houkin K: Surgical management of the recurrence and regrowth of craniopharyngiomas. J Neurosurg 103:224-232, 2005

23. Mortini P, Losa M, Pozzobon G, Barzaghi R, Riva M, Acerno $S$, et al: Neurosurgical treatment of craniopharyngioma in adults and children: early and long-term results in a large case series. J Neurosurg 114:1350-1359, 2011

24. Munari C, Landre E, Musolino A, Turak B, Habert MO, Chodkiewicz JP: Long term results of stereotactic endocavitary beta irradiation of craniopharyngioma cysts. J Neurosurg Sci 33:99-105, 1989

25. Patel MR, Taylor RJ, Hackman TG, Germanwala AV, SasakiAdams D, Ewend MG, et al: Beyond the nasoseptal flap: outcomes and pearls with secondary flaps in endoscopic endonasal skull base reconstruction. Laryngoscope 124:846-852, 2014

26. Prieto R, Pascual JM, Subhi-Issa I, Jorquera M, Yus M, Martínez R: Predictive factors for craniopharyngioma recurrence: a systematic review and illustrative case report of a rapid recurrence. World Neurosurg 79:733-749, 2013

27. Regine WF, Mohiuddin M, Kramer S: Long-term results of pediatric and adult craniopharyngiomas treated with combined surgery and radiation. Radiother Oncol 27:13-21, 1993

28. Schoenfeld A, Pekmezci M, Barnes MJ, Tihan T, Gupta N, Lamborn KR, et al: The superiority of conservative resection and adjuvant radiation for craniopharyngiomas. $\mathbf{J}$ Neurooncol 108:133-139, 2012 
29. Solanki SP, Sama A, Robertson IJ: Endoscopic transnasal external fistulation in recurrent cystic subdiaphragmatic craniopharyngioma: a novel technique. J Neurosurg Pediatr 17:141-146, 2016

30. Spaziante R, De Divitiis E, Irace C, Cappabianca P, Caputi $\mathrm{F}$ : Management of primary or recurring grossly cystic craniopharyngiomas by means of draining systems. Topic review and 6 case reports. Acta Neurochir (Wien) 97:95106,1989

31. Stagno V, Cappabianca P: Which side tips the scale for recurrent craniopharyngiomas? World Neurosurg 79:647648,2013

32. Šteňo J, Bízik I, Šteňo A, Matejčík V: Recurrent craniopharyngiomas in children and adults: long-term recurrence rate and management. Acta Neurochir (Wien) 156:113-122, 2014

33. Wu P, Li Z, Liu C, Ouyang J, Zhong S: The posterior pedicled inferior turbinate-nasoseptal flap: a potential combined flap for skull base reconstruction. Surg Radiol Anat 38:187-194, 2016

34. Zanation AM, Snyderman CH, Carrau RL, Kassam AB, Gardner PA, Prevedello DM: Minimally invasive endoscopic pericranial flap: a new method for endonasal skull base reconstruction. Laryngoscope 119:13-18, 2009

\section{Disclosures}

The authors report no conflict of interest concerning the materials or methods used in this study or the findings specified in this paper.

\section{Author Contributions}

Conception and design: Zadeh, Gentili. Acquisition of data: Turel, Tsermoulas, Gonen. Analysis and interpretation of data: Turel, Tsermoulas, Gonen. Drafting the article: Turel. Critically revising the article: Tsermoulas, Gonen, Klironomos, Almeida, Zadeh, Gentili. Reviewed submitted version of manuscript: all authors. Approved the final version of the manuscript on behalf of all authors: Turel.

\section{Correspondence}

Mazda K. Turel, Division of Neurosurgery, Toronto Western Hospital, 399 Bathurst St., Toronto, ON M5T 2S8, Canada. email: mazdaturel@gmail.com. 